\title{
Innovatory designs for ducted wind turbines
}

\author{
Ssu-Yuan $\mathrm{Hu}^{\mathrm{a}}$, Jung-Ho Cheng ${ }^{\mathrm{b}, *}$ \\ ${ }^{a}$ Industrial Technology Research Institute, No. 195 Chung-Hsing Road, Section 4, Chutung 310, Taiwan, ROC \\ ${ }^{\mathrm{b}}$ Department of Mechanical Engineering, National Taiwan University, No. 1 Roosevelt Road, Section 4, Taipei 106, Taiwan, ROC
}

Received 4 May 2007; accepted 15 August 2007

Available online 1 November 2007

\begin{abstract}
Designs for conventional ducted wind turbines usually include a large inlet for more absorption of the airflow. However, the most efficient solution should be increasing the speed of wind. In this paper, a bucket-shape ducted wind turbine is proposed and studies show that a sucking effect can be produced according to the Bernoulli's principle, and this significantly increases the wind speed inside the duct and substantially enhances the efficiency of the wind turbine. Moreover, the geometry of the duct is optimized by the combination of an improved complex algorithm, an object-oriented optimizing program interface, and simulations by CFD software. According to the analyses, the optimal shape for the interior of the duct appears to be an unconventional nozzle, which extends the range of wind speed by $60 \%$.

Based on this bucket duct equipped with the optimal nozzle, we have practically designed and constructed a wind power generator. The results of the field tests show that the proposed ducted turbine does improve the flow around the generator and thus increase its power extraction efficiency by about $80 \%$.
\end{abstract}

(C) 2007 Elsevier Ltd. All rights reserved.

Keywords: Ducted wind turbines; Optimization; CFD; Wind power; Renewable energy

\section{Introduction}

It is a general perception that promoting substitutive energy sources to replace fossil fuels is becoming more and more urgent, since the concern for global energy crisis has been gaining substantial momentum in the past few decades. World trend also suggests that renewable energy technologies offer the most promising candidate in the premonition of the imminent depletion of petroleum. Among all renewable energy of different styles, wind energy possesses many advantages such as extensive distribution, high efficiency, low cost, low maintenance, environmental friendliness etc. and it is the most popularized and potentially applicable type of green energy. Therefore, people have long been seeking designs of enhancements to the conventional wind turbines, and one of the most assuring proposals is the ducted wind turbine.

The idea of ducted wind turbines is to construct a funnel or a conduit around a conventional wind turbine to adjust

\footnotetext{
*Corresponding author. Tel.: + 886223622191 ; fax: + 886223631755 .

E-mail address: jhcheng@ntu.edu.tw (J.-H Cheng).
}

the airflow in order to raise the power extraction efficiency. In its early stage of development, the design usually featured a large inlet to absorb more wind, and became impractical since the duct usually costs more than the turbine itself. However, smaller and cheaper designs are continuously introduced along with the rapid evolvement of aerodynamics, and they mostly bear the common function to induce more velocity rather than more airflow, which results from the fact that the dynamic energy of the wind is proportional to the cubic of its velocity [1-6].

Therefore, the aim of this paper is to explore the relations between duct shapes and wind velocities, to arrange design parameters of duct geometries for optimal flow acceleration, and to raise the efficiency of ducted wind turbines. The resulting design will be practically tested to demonstrate its feasibility.

\section{Numerical model}

Experimental tests over all kinds of design proposals would be undoubtedly too costly, so a computational fluid 
dynamics (CFD) model of the basic type of the proposed duct was first constructed for simulation, and Fig. 1 illustrates the fundamental concept of the new wind duct along with initial design parameters. The idea of the configuration of the duct is to guide the airflow into the inlet and across the outlet simultaneously so that a lowpressure zone develops alongside the outlet to create a suction effect which accelerates the airflow inside the duct. To observe the connections between duct geometry and wind speeds, CFD simulations give us a clear and comprehensible picture about the pressure and velocity distributions within the duct.

According to the dimensions of the initial design, a corresponding CFD mesh was constructed using brick elements solely, and only elements on the boundary are shown in Fig. 2 for the sake of easy observation. The solid frame represents the inlet boundary condition of a $5 \mathrm{~m} / \mathrm{s}$ inflow normal to the surface, while the dashed represents the symmetry plane. The ground and the duct in light gray are set to wall condition, and the dark gray parts indicate free outlet condition. The model was established and meshed by the CAD software I-DEAS v9.0, and subsequent simulations were carried out by the CFD software Fluent v6.1.

As the wind turbine is installed inside the duct brim, which is indicated as part A in Fig. 4, all transverse sections of the meshes inside the ring are marked for output and monitoring, and the variation of the energy flux passing through these sections is recorded for comparison (Fig. 3). Convergence tests on four models of different mesh sizes (7082, 24,440, 77,655 and 168,320 elements) describing the same boundary conditions yield the curve in Fig. 5, which demonstrates the simulated maximal energy flux. Since the scale of the models is not of significance, the results were normalized with respect to that of the most elements. This curve suggests that approximately 25,000 elements are sufficient to produce a converging simulation. All subsequent adjustments or modifications will be added with respect to this basic model which serves as a reference for comparison.

\section{Optimization}

Since the correlations between duct geometries and resulting flow fields are so complicated and perplexing, it

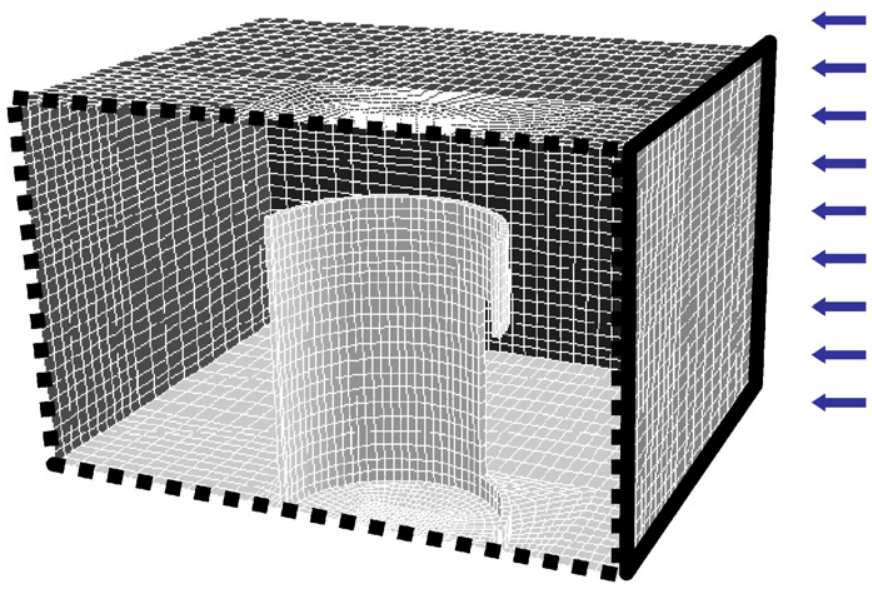

Fig. 2. Geometry and boundary of the CFD mesh.
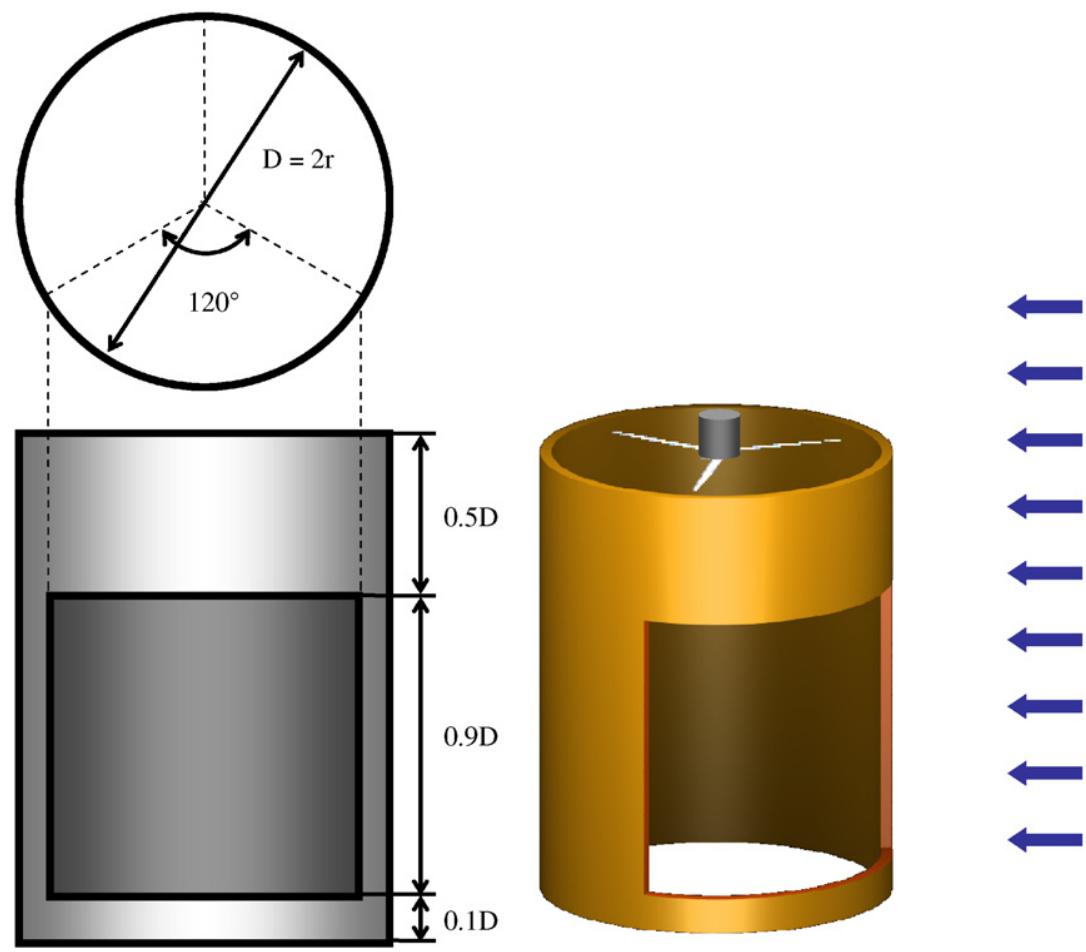

Fig. 1. Concept of the innovative duct. 


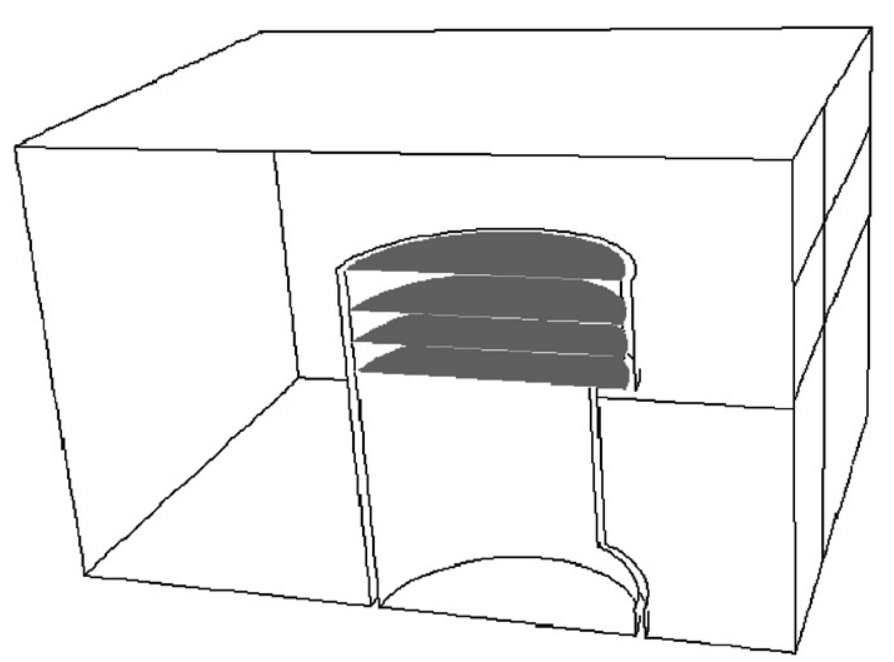

Fig. 3. Sections marked for monitoring.

a

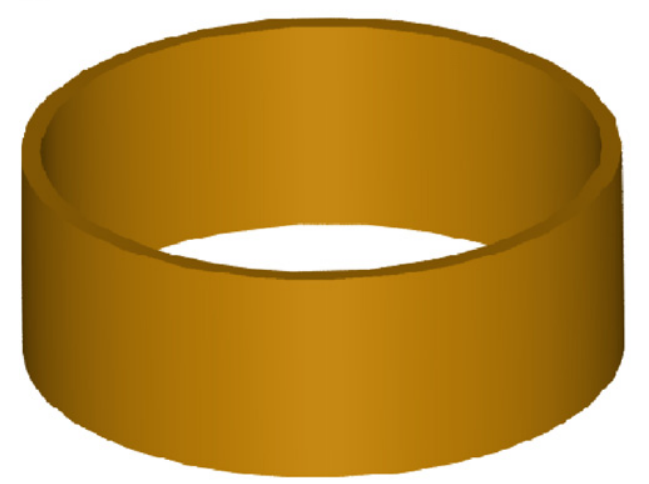

b

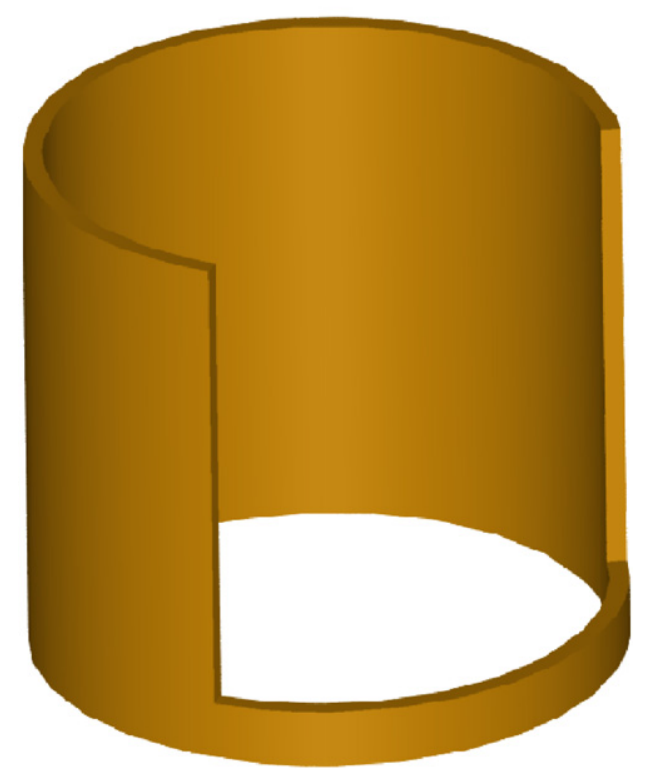

Fig. 4. Upper and lower parts of the duct.

is too difficult to derive the analytical forms of their connections. Therefore, the most straightforward and efficient way to search for the most applicable shape is through optimization design. Because an analytical form

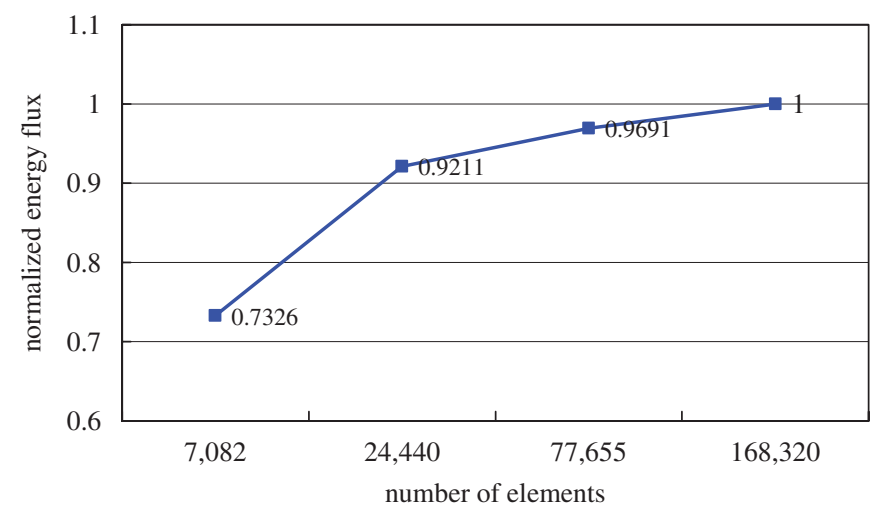

Fig. 5. Convergence test of the CFD mesh.

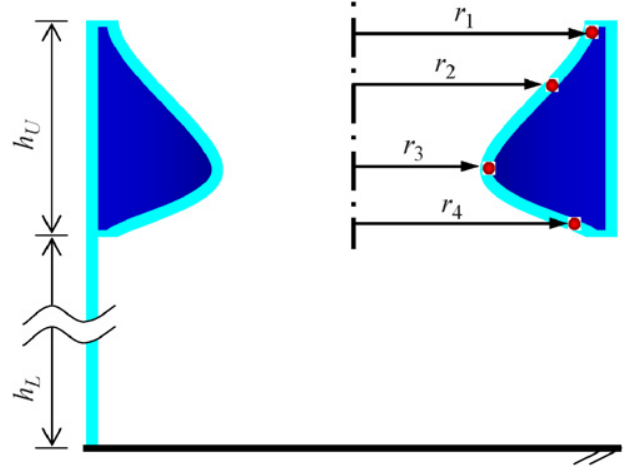

Fig. 6. Parametrization of the nozzle.

for the objective function is unavailable due to the adoption of CFD simulations, the optimization process utilized a heuristic optimizing method together with a general programming interface to accommodate arbitrary algorithms and engineering software, and the collaboration of assorted programs eventuated in an optimal shape for subsequent duct design.

\subsection{Objective function}

The duct can be divided into two parts, as shown in Fig. 4. The upper part A is axisymmetric, while the lower part B is not. Therefore, part A stands an obviously better target for shape optimization. One single spline would suffice to describe arbitrary shape of the duct interior, and the resulting flow velocity or dynamic energy is merely a function of the coordinates defining this spline. With the illustration of Fig. 6, the objective function can be defined as

$$
f\left(r_{i}\right)=\operatorname{Max}(W)
$$

subject to $0<r_{i}<R_{i}(i=1-4)$,

where $r_{i}$ are the radial coordinates of the four control points located according to Chebychev distribution in the axial direction; $R_{i}$ are design space of $r_{i}$, and are all set to 
the inner diameter of part $\mathrm{A}$; $W$ represents the recorded dynamic energy of the airflow passing through every section in Fig. 3; $\operatorname{Max}(W)$ means the maximum of $W$ recorded among all sections, and the section on which $\operatorname{Max}(W)$ registers indicates the elevation for the installation of the wind turbine. Therefore, Eq. (1) defines the maximal energy as a function of $r_{i}$, which is to be optimized. It should be clarified that the maximum referred stands for the statistical extreme among the sections, not the optimized results. Subsequent optimal design involves further searching for the maximum of $\operatorname{Max}(W)$ with respect to $r_{i}$.

\subsection{Optimizing algorithm and programming}

A heuristic algorithm termed the Raplex method was adopted to solve this optimization problem. Raplex method is a modification to the complex method suggested by M. Box, and its improvements include unlocking mechanisms, higher evaluation efficiency and robustness of convergence [7].

The key to the integration of the optimizing algorithm, objective function and CFD simulations is to set up an interfacing environment, where independent codes can communicate and share data through well-organized channels [8]. This paper adopts VB 6.0 as a programming tool since the embedded syntax parser versatilely allows post-compilation function definition, and Fig. 7 reveals the interface structure of the object-oriented optimization program. When a new set of $r_{i}$ is suggested by the Raplex algorithm, the interface module generates a new mesh describing the corresponding duct shape by adjusting nodal coordinates of the basic model in Fig. 2. Then the module calls Fluent software for simulation computations, keeps monitoring the solver status and retrieves the output data after the calculations are completed. Then the essential results are converted to proper format and transferred back to the algorithm as the evaluation of the objective function, and the algorithm goes back to iterating and searching for

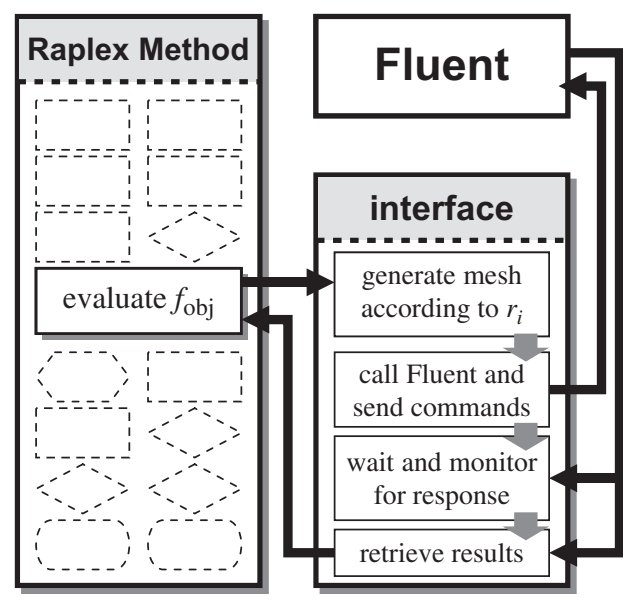

Fig. 7. Data flow of the interface program. better suggestions of $r_{i}$ coordinates until it reaches a converged optimum [9].

\subsection{Results and discussion}

The nozzle shapes in Fig. 8 demonstrate the most representative optimal results for the duct design of different $h_{\mathrm{U}} / h_{\mathrm{L}}$ ratios. Unlike traditional nozzles, the slopes at both ends of the optimal profile tend to incline towards the radial direction, which coincides with the direction of particle path lines, and this makes a plausible and reasonable solution to the problem. Simulations on the optimal shape for $h_{\mathrm{U}}=0.5 \mathrm{D}$ and $h_{\mathrm{L}}=1.0 D$ reveal that the duct accelerates the inflow by a factor of approximately 2.1, as shown in Fig. 9.

Besides, there appears to be a lower limit for the throat size. Despite the fact that smaller sections result in faster airflow, the corresponding resistance increases as well.

a

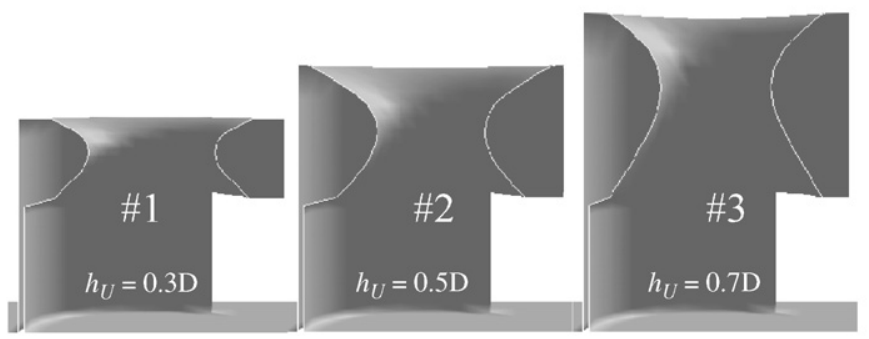

b

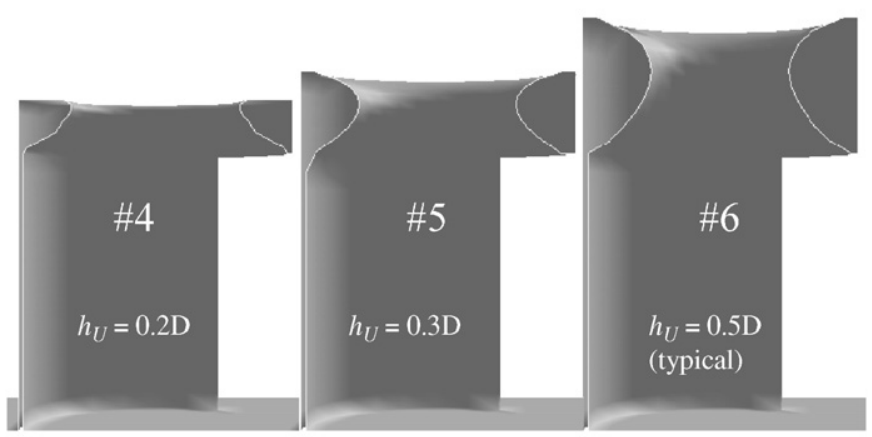

C

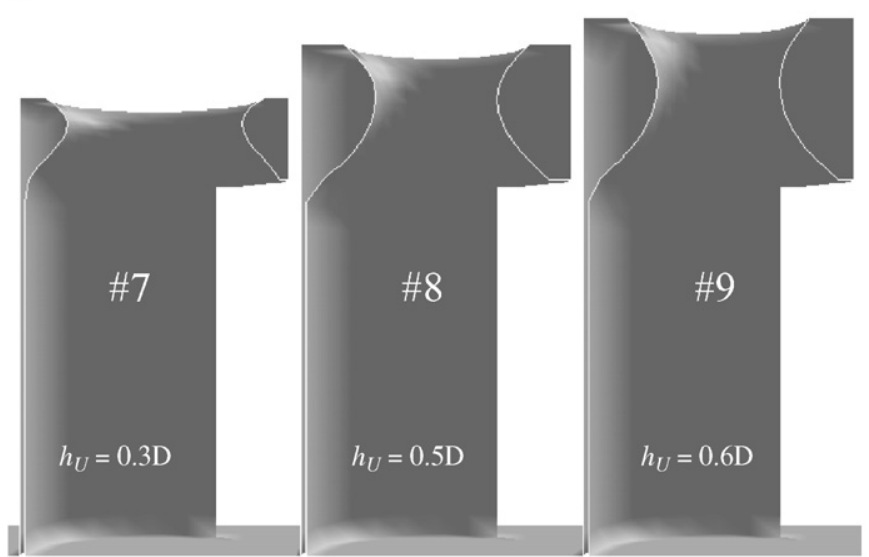

Fig. 8. Optimal duct shapes: (a) $h_{\mathrm{L}}=0.5 D$, (b) $h_{\mathrm{L}}=1.0 D$ and (c) $h_{\mathrm{L}}=1.4 D$. 


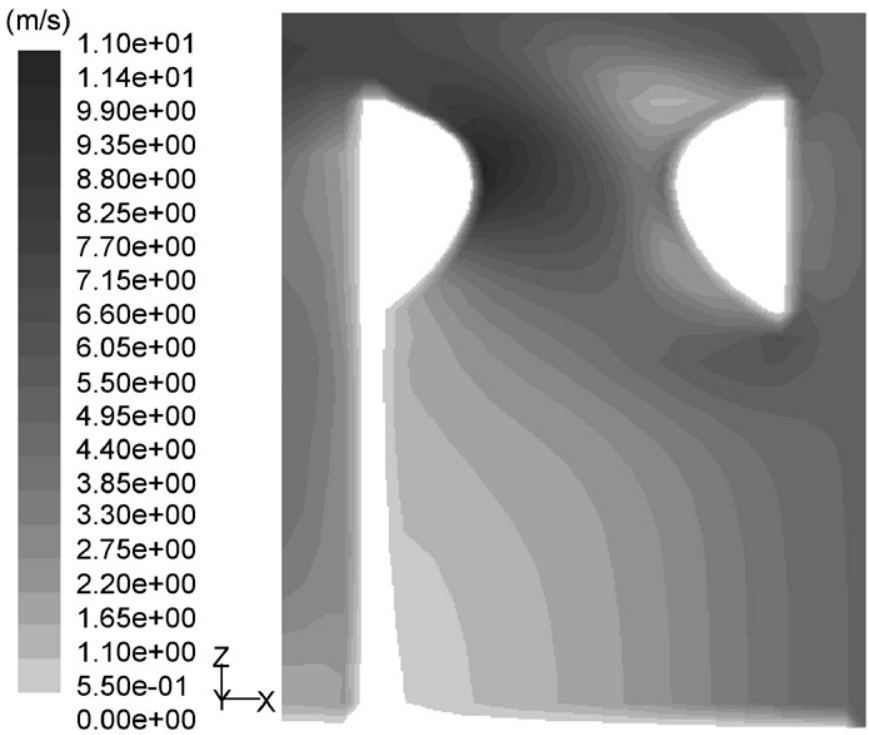

Fig. 9. CFD simulated velocity field of the optimized typical duct.

In Fig. 8, the lower limit of the optimal section diameter goes down to approximately $50 \%$ of that of the original duct. Moreover, this limit is independent of $h_{\mathrm{L}}$, as long as $h_{\mathrm{U}}$ is large enough to accommodate the transition of the profile.

To summarize on the optimal duct shapes, one should notice that all optimal shapes are geometrically similar in spite of the different $h_{\mathrm{U}} / h_{\mathrm{L}}$ ratios. This observation indicates that designs for a new $h_{\mathrm{U}} / h_{\mathrm{L}}$ ratio do not require additional optimization processes, for they can be derived from known optimal shapes by scaling them to the desired dimensions. This conclusion significantly facilitates subsequent design processes because time and computing resources can be substantially reduced.

\section{Experiments}

Combined with the wind turbine, the skeleton structure and the optimized nozzle shape for the duct design, a prototype of the ducted wind turbine was designed and manufactured. This turbine consists of a stationary part and a rotary part, and the completed installation was tested for its practicability.

\subsection{Assembly}

The key dimensions of the structure are decided as follows: both the overall height and diameter of the whole structure are $3.0 \mathrm{~m}$, and the nozzle height is $40 \mathrm{~cm}$. We adopt Southwest Windpower's H40 turbine for the experiment, and its diameter is $2.1 \mathrm{~m}$. The reason why the nozzle height is relatively small is that the duct of the turbine is to be fabricated by $\mathrm{CNC}$, and the nozzle design is thus economically and dimensionally compromised due to machine capabilities and cost considerations. As a result, $h_{\mathrm{U}}$ for the experimental assembly is approximately $0.13 D$ rather than $0.5 D$ of the typical duct. As Fig. 10 illustrates,

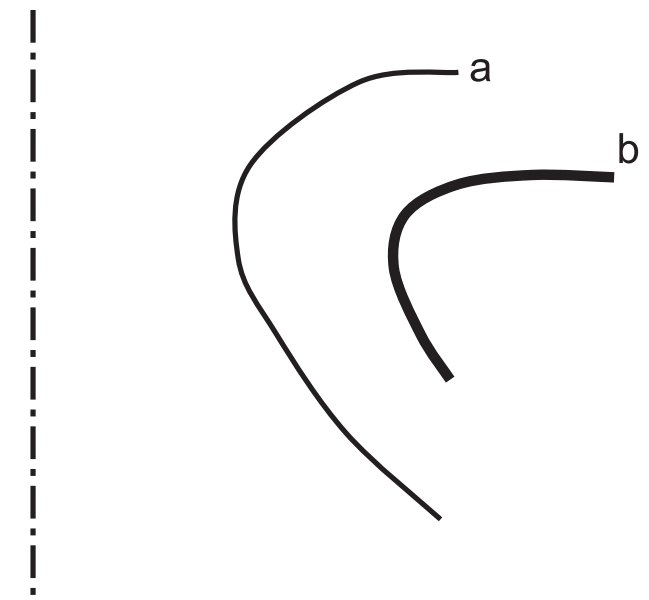

Fig. 10. Nozzle profiles: (a) optimal results of the typical duct and (b) adopted profile for the experimental setup.

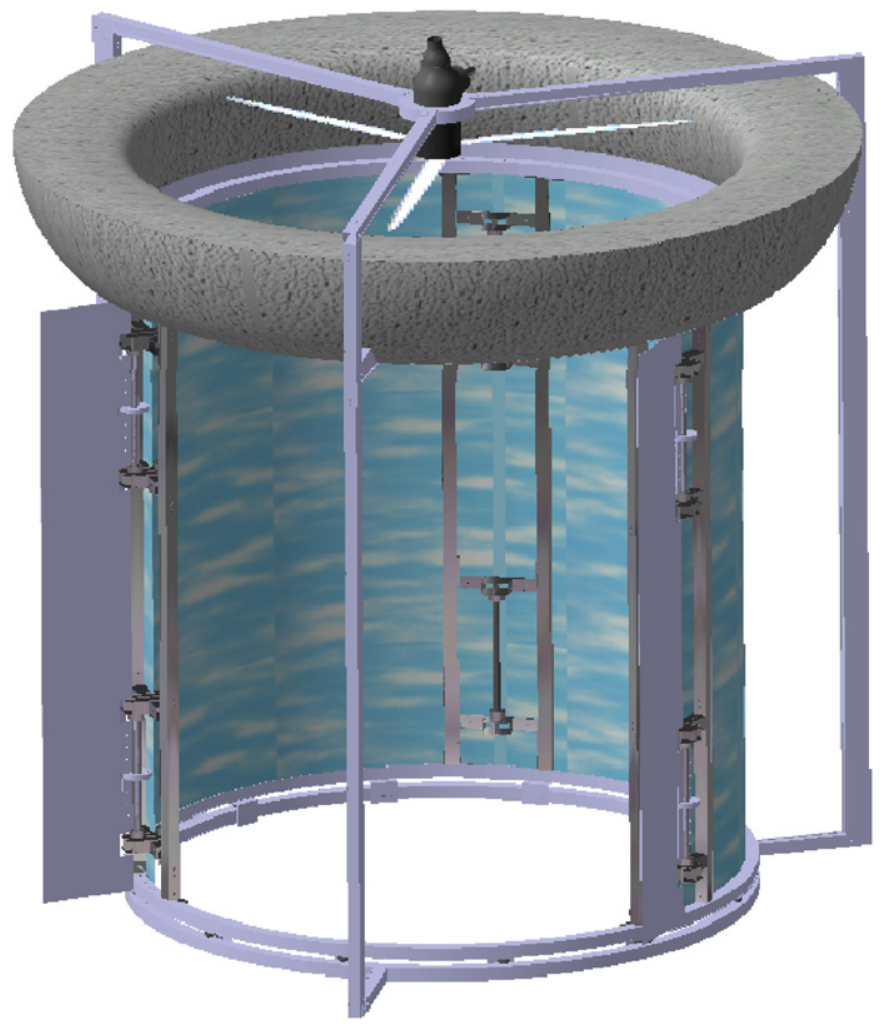

Fig. 11. CAD design of the proposed turbine.

the nozzle shape is acquired from case \#4 in Fig. 8, since it is concluded that any new profile can be derived by scaling known optimal results. Despite the slight difference between the experimental and typical designs, their profiles are still geometrically similar.

The stationary part consists of the turbine, the nozzle and their supporting structure, while the rotary part includes a bucket with a $120^{\circ}$ window and a set of rollers providing the rotating abilities for the bucket to aim its inlet toward the wind. All the features mentioned above unite as the final design of the ducted wind turbine, as Fig. 11 depicts. A corresponding CFD model was 
established to verify the final design, and Fig. 12 demonstrates its acceleration ability. Due to the compromise to the manufacturing limitations, the wind speed is increased by a factor of 1.6 rather than 2.1 of the optimal nozzle in Fig. 9. The dynamic energy of the passing airflow is raised by $20 \%$ with respect to a naked turbine, and it should also be noticed that the outer halves of the blades intercept most of the dynamic energy.

One should perceive that passing energy does not necessarily mean captured energy, since air near the root of blades is usually wasted due to stall [10]. To explore the differences, effective power exerted on the blades is approximated by the illustrations in Fig. 13. Assuming that the overall power of air passing through a traditional conventional turbine is $W$ (which evenly is exerted on the blade) and that of the proposed duct is $1.2 \mathrm{~W}$ (which acts only on the outer half of the blades), the torque of the conventional turbine can be estimated as

$\int_{0}^{R} \frac{c k W}{R} r \mathrm{~d} r=0.5 c k R W$,

while that of the proposed turbine is

$\int_{R / 2}^{R} \frac{1.2 c k W}{R / 2} r \mathrm{~d} r=0.9 c k R W$

where $R$ is the blade length, $c$ is the power extraction efficiency of the blade element, and $k$ is the ratio of the exerted aerodynamic force to the passing dynamic energy of the blade element [11]. Therefore, the power extraction efficiency of the ducted wind turbine exceeds that of the conventional turbine by about $80 \%$.

Since the final design of the duct is verified, the detailed mechanisms are designed and assembled on the CAD software CATIA v5r10, and the whole installation is pragmatically set up as shown in Fig. 14.

\subsection{Experiments}

The aim of the experiment is to confirm the consistency of the accelerating ability of the duct between CFD simulations and actual situations. Two sets of anemometers are fixed at designated positions in Fig. 15, where set $\mathrm{A}$ records the variation of accelerated velocities, and set $\mathrm{B}$ records that of the inflow. The results from both anemometers are plotted in Fig. 16, where $x$-axis represents values of anemometer $\mathrm{B}$, and $y$-axis of anemometer $\mathrm{A}$. Outlet speeds under $3 \mathrm{~m} / \mathrm{s}$ or inlet speeds under $1.5 \mathrm{~m} / \mathrm{s}$ are discarded beforehand since the startup velocity of the adopted $\mathrm{H} 40$ turbine is about $3.4 \mathrm{~m} / \mathrm{s}$, so lower speeds representing erratic winds should be neglected for better assessment of duct performance. The slopes from origin to these points imply the factor of acceleration, which ranges from 1.30 to 1.93 . The averaged value is 1.62 , which concurs with the results from Fig. 12.

\subsection{Discussion}

Due to the fact that the acquisition of a perfect site for wind power generation is difficult and costly, the above

a

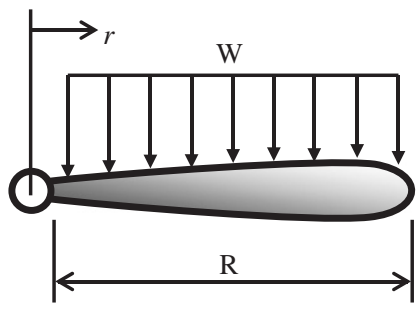

Fig. 13. Power extraction approximation of (a) traditional and (b) proposed turbines.

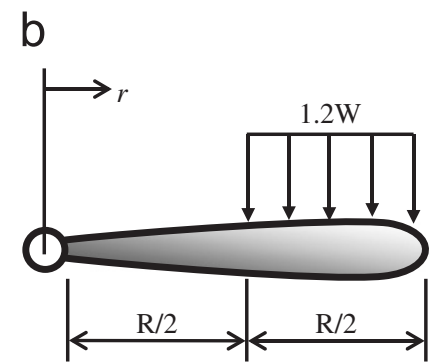

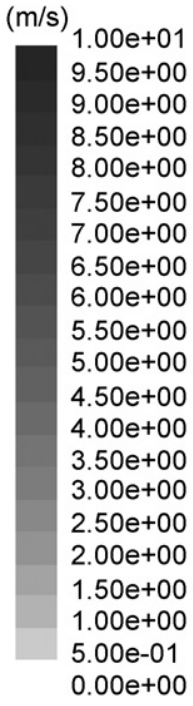

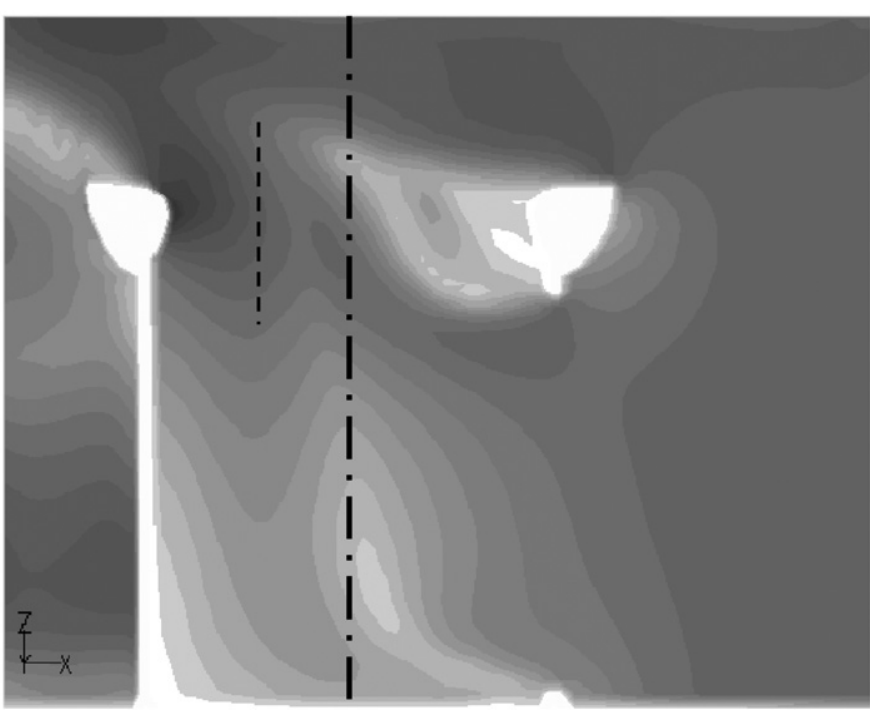

Fig. 12. CFD simulated velocity field of the prototype. 

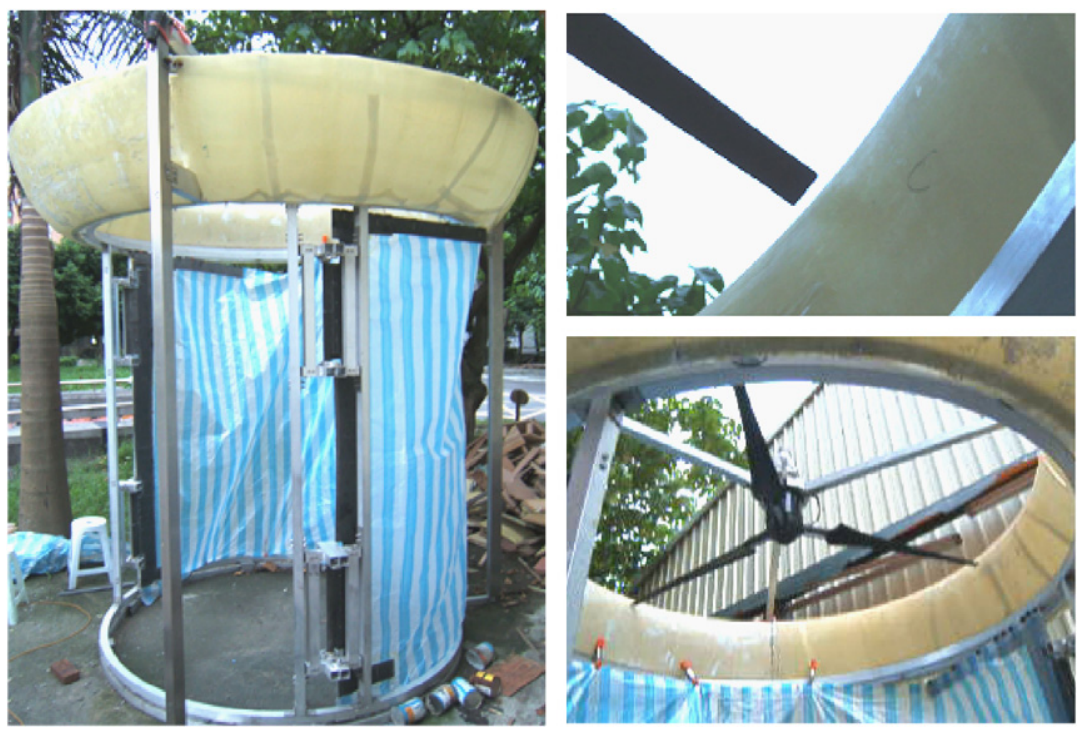

Fig. 14. Actual installation of the ducted wind turbine.

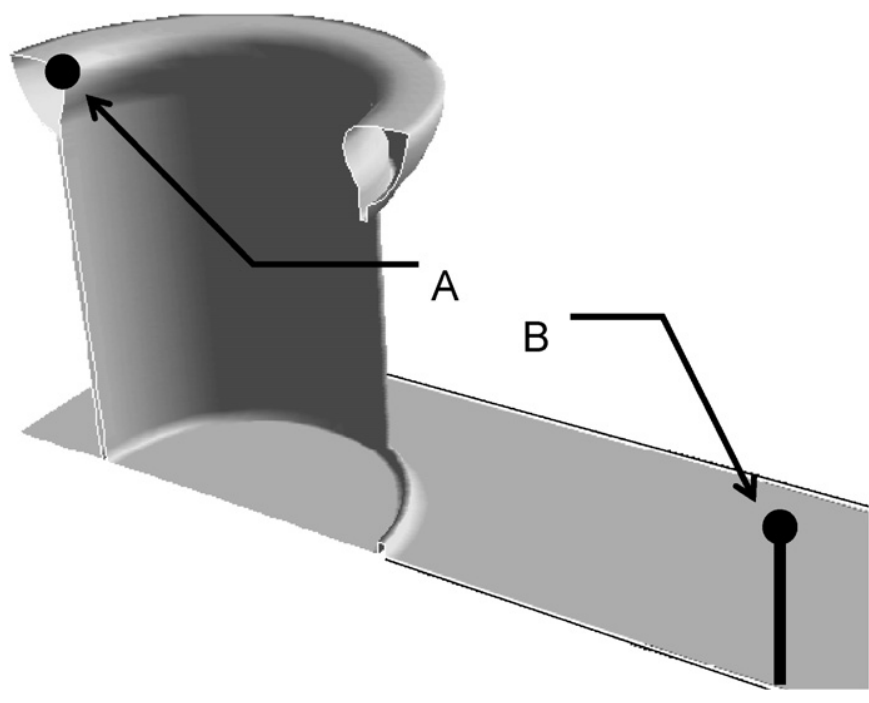

Fig. 15. Anemometer setup for the experiment.

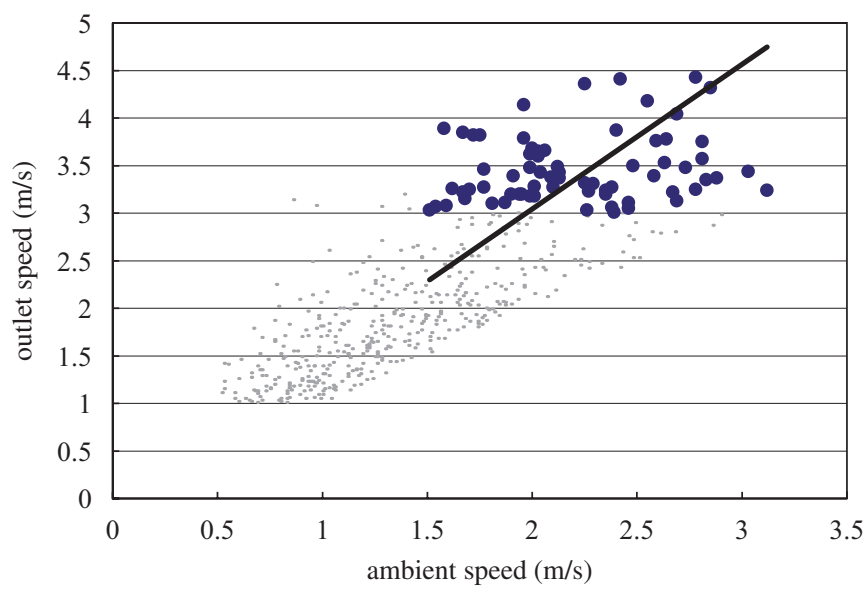

Fig. 16. Experimental results. experiments were conducted on the campus where the wind distributions were capricious and unfavorable for effective power utilization, so the distribution of recorded wind speeds is very unstable. Moreover, one point should be emphasized that all ambient velocities recorded in Fig. 16 range below $3.4 \mathrm{~m} / \mathrm{s}$, which means that the adopted turbine could not even start up under naked conditions, not to mention operate. However, with the aid of the proposed duct, extra energy can be extracted to raise the efficiency of the turbine.

The capital cost of the modified turbine is also of interest. In the presented case, the retail price of the adopted $\mathrm{H} 40$ turbine is $\$ 2156.25$ (USD), and its compatible towers cost more than $\$ 499.0$, which is about $23 \%$ of the turbine price. The cost of materials and production of a single duct is about $\$ 1200$, which is equivalent to $55 \%$ of the turbine price. Assuming all other conditions are identical, the proposed ducted turbine costs $26.4 \%$ more than a naked conventional turbine, while it captures $60-80 \%$ more energy. Moreover, since the duct is just an experimental prototype, the efficiency will undoubtedly be increased with future modifications, and the cost can also be further reduced by mass production. This makes quite a reasonable and economically competitive solution to a new duct design.

\section{Conclusions}

In this paper, a CFD model for the presented turbine was first constructed for analysis. Besides, an interface program capable of generating modified meshes with assigned duct shapes and communicating with CFD software was also compiled for efficient data management. Combined with optimization process and CAD, a brand new ducted wind turbine was designed, manufactured and 
tested, and the results demonstrate significant improvement over conventional turbines.

Moreover, experimental results also reveal further ways of explorations for future researches. After the successful optimization to the inner profile of the nozzle, the outer surface may need to be tuned as well for better efficiency. Also, a non-axisymmetric nozzle design may also be of consideration for sites with a prevailing wind direction. Besides, previous proposals by other researchers can also be introduced to this turbine for additional refinements. For example, a steering aerofoil in front of the blades could result in higher energy output without obstructing the original design [12].

Finally, more accurate experiments such as wind tunnel tests on the duct should be conducted to investigate more detailed accelerating behaviors of the nozzle in the hope of providing a much broader scope for related researches about performances of ducted wind turbines.

\section{Acknowledgment}

The authors wish to acknowledge the financial support by the National Science Council of Republic of China through the Project NSC 94-2212-E-002-044.

\section{References}

[1] Bet F, Grassmann H. Upgrading conventional wind turbines. Renew Energy 2003;28:71-8.

[2] Dannecker RKW, Grant AD. Investigations of a building-integrated ducted wind turbine module. Wind Energy 2002;5:53-71.

[3] Frankovic B, Vrsalovic I. New high profitable wind turbines. Renew Energy 2001;24:491-9.

[4] Lawn CJ. Optimization of the power output from ducted turbines. J Power Energy 2003;217:107-17.

[5] Grassmann H, Bet F, Ceschia M, Ganis ML. On the physics of partially static turbines. Renew Energy 2003;29:491-9.

[6] Grassmann H, Bet F, Cabras G, Ceschia M, Cobai D, DelPapa C. A partially static turbine-first experimental results. Renew Energy 2003;28:1779-85.

[7] Hu S, Cheng J. Development of the unlocking mechanisms for the complex method. Comput Struct 2005;83:1991-2002.

[8] Hu S, Cheng J. Development of an object-oriented optimization software for industrial utilization. In: Proceedings of the fourth conference of OPTDES, 2004.

[9] $\mathrm{Hu} \mathrm{S}$, Cheng J. An innovative design of ducted wind turbine. In: Proceedings of world renewable energy congress IX, 2006.

[10] Wright AK, Wood DH. The starting and low wind speed behaviour of a small horizontal axis wind turbine. J Wind Eng Ind Aerodyn 2004;92:1265-79.

[11] Burton T, Sharpe D, Jenkins N, Bossanyi E. Wind energy handbook. Wiley; 2001. p. 59-65.

[12] Varol A, Varol Y. Increasing the efficiency of wind turbines. J Wind Eng Ind Aerodyn 2001;89:809-15. 